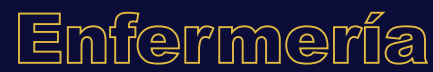

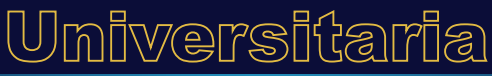

\section{Uso de Tai Chi para la salud del adulto mayor: revisión bibliográfica}

\author{
Use of Tai Chi for the health of the \\ elderly: A bibliographic review
}

\section{Uso do Tai Chi para a saúde de idosos: uma revisão bibliográfica}

O. Cruz-Cartas ${ }^{\mathrm{a} 1}$, M.L. García-Campos ${ }^{\mathrm{b} 1,2^{*} \text {, }}$ V. Beltrán-Campos ${ }^{\mathrm{c}}$, X.S. Ramírez-Gómez ${ }^{\mathrm{d} 1}$, M.E. Patiño-López ${ }^{\mathrm{e}}$, S.N. Jiménez-García ${ }^{\mathrm{f} 1}$

ORCID

${ }^{\mathrm{a}} 0000-0003-1588-2475$

d0000-0002-4400-480X

b0000-0002-5406-5286

e $0000-0001-7256-3759$

c0000-0001-8925-811X

${ }^{\mathrm{f}} 00000-0003-2713-4693$

${ }^{1}$ Universidad de Guanajuato, División de Ciencias de la Salud e Ingenierías, Departamento de Enfermería Clínica, Campus Celaya-Salvatierra, Celaya, Guanajuato, México

${ }^{2}$ Instituto Mexicano del Seguro Social, Hospital General de Zona No. 4, Celaya, Guanajuato, México

Recibido: 13 diciembre 2019

Aceptado: 05 marzo 2020

RESUMEN

Introducción: La población de adultos mayores está incrementando y se observa la necesidad emergente de implementar estrategias dirigidas a recuperar o conservar su salud para que puedan gozar de una vejez plena. Técnicas como el Tai Chi podrían contribuir, ya que han mostrado tener múltiples beneficios para quien la práctica, tales como mejoras en la concentración, memoria, marcha, equilibrio, reducción del riesgo de caídas, fortalecimiento del sistema cardiovascular, reducción del estrés y la depresión, entre otros beneficios.

Objetivo: Analizar la evidencia científica del uso del Tai Chi para conservar la salud del adulto mayor.

* Autora para correspondencia. Correo electrónico: lulu.garcia@ugto.mx https://doi.org/10.22201/eneo.23958421e.2021.2.805

1665-7063/@ 2021 Universidad Nacional Autónoma de México, Escuela Nacional de Enfermería y Obstetricia. Este es un artículo Open Access bajo la licencia CC BY-NC-ND (http://creativecommons.org/licenses/by-nc-nd/4.o/). 
Desarrollo: Revisión bibliográfica realizada durante el periodo 2013-2019 en distintas bases de datos como: Pubmed, Dialnet, SciELO, LILACS, ScienceDirect y Google Académico. Se utilizaron las palabras Tai Chi Chuan, Tai Ji Quan, Tai Chi. Aplicando los criterios de elegibilidad se seleccionaron 29 artículos.

Resultados: Gran porcentaje de los artículos revisados estudian la atención de síntomas físicos que deterioran la movilidad del adulto mayor, sin embargo, también se encontraron investigaciones dirigidas a la parte psicológica, como el estrés, la ansiedad, el insomnio, la depresión y el enfado-tensión, en las cuales la práctica del Tai Chi demostró mejoría.

Conclusiones: El Tai Chi es una terapia alternativa para prevenir y conservar la salud del adulto mayor, fácil de aprender y de bajo costo. En la revisión bibliográfica realizada no se reportan reacciones adversas durante ni después de la práctica de la técnica; el profesional de enfermería puede recomendarla ampliamente para ser implementada como un cuidado dirigido al adulto mayor.

Palabras clave: Tai Chi; tai ji; terapia por ejercicio/métodos; salud; adulto mayor; anciano; México.

\section{ABSTRACT}

Introduction: The population of older adults is increasing and an emergent need to implement strategies aimed at recovering or maintaining their health is observed. In this sense, techniques such as Tai Chi could contribute because they have demonstrated bringing diverse benefits for those who practice them, including improvements in the concentration, memory, gait, and equilibrium, a reduction in the risk of falls, a strengthening of the cardiovascular system, and a reduction of stress and depression, among other benefits.

Objective: To analyze the scientific evidence on the use of Tai Chi as a health promoter and preserver among older adults.

Development: This bibliographic review was conducted during 2013-2019 on the Pubmed, Dialnet, SciELO, LILACS, ScienceDirect and Academic Google databases. The keywords used were Tai Chi Chuan, Tai Ji Quan, and Tai Chi.After filtering through the eligibility criteria, 29 articles were selected for the review.

Results: An important percentage of the articles were focused on the attention to physical symptoms which impair the mobility of older adults; nevertheless, some studies addressed psychological issues such as stress, anxiety, sleep disorders, depression, and anger-tension, as well. In these studies, the practice of Tai Chi was associated with health improvements among older adults.

Conclusions: Tai Chi is an alternative therapy to promote and maintain health among older adults. This technique is easy to learn, and its practice is not expensive. No adverse reactions during or after the practice of this technique were reported in the studies of this literature review. Therefore, the nursing professional can recommend Tai Chi practice to be implemented as a complementary healthcare measure for older adults.

Keywords: Tai Chi; tai ji; exercise therapy/methods; health; elderly; aged; Mexico.

\section{RESUMO}

Introdução: A população de idosos está aumentando e observa-se a necessidade emergente de implementação de estratégias que visem a recuperação ou preservação da saúde para 
que possam desfrutar de uma velhice plena. Técnicas como o Tai Chi podem contribuir, dado que têm se mostrado múltiplos benefícios para quem o pratica, tais como melhora na concentração, memória, marcha, equilíbrio, redução do risco de quedas, fortalecimento do sistema cardiovascular, redução de estresse e depressão, entre outros benefícios.

Objetivo: Analisar a evidência científica do uso do Tai Chi na preservação da saúde de idosos. Desenvolvimento: Revisão bibliográfica realizada no período 2013-2019 em diferentes bases de dados como: Pubmed, Dialnet, SciELO, LILACS, ScienceDirect e Google Academic. Foram utilizadas as palavras Tai Chi Chuan, Tai Ji Quan, Tai Chi. Aplicando os critérios de elegibilidade, foram selecionados 29 artigos.

Resultados: Grande porcentagem dos artigos revisados estuda a atenção aos sintomas físicos que prejudicam a mobilidade do idoso, porém, as pesquisas também foram direcionadas à parte psicológica, como estresse, ansiedade, insônia, depressão e raiva-tensão, em que a prática do Tai Chi demonstrou melhora.

Conclusões: O Tai Chi é uma terapia alternativa para prevenir e preservar a saúde do idoso, de fácil aprendizado e de baixo custo. Na revisão bibliográfica realizada, não foram relatadas reações adversas durante ou após a prática da técnica; o profissional de enfermagem pode recomendar veementemente que seja implementada como cuidado ao idoso.

Palavras chave: Tai Chi; tai ji; terapia por exercício/métodos; saúde; idoso; velho; México.

\section{INTRODUCCIÓN}

El incremento de la población de adultos mayores es una realidad en todo el mundo; ante eso, la Organización Mundial de la Salud (OMS) prevé que este grupo de edad corresponderá a 21 \% de la población para el año $2050^{1}$ a nivel nacional e internacional. Por su parte, en México, el Consejo Nacional de Población (CONAPO) también predice un aumento de este grupo de edad de 24.4 millones para el año $2050^{2}$.

El aumento de la esperanza de vida es un logro para los países en desarrollo y en vías de desarrollo33. Con ello, se prevé que acrecentará la demanda en los servicios de salud, como consulta médica, horas de estancia hospitalaria, cirugías, medicamentos y reingreso al hospital derivado de alguna complicación. Esto también impactará en el sustento económico de las familias, pues en algún punto se verán en la necesidad de cubrir el costo de personal de cuidados para el familiar enfermo, además de pagar el material requerido para dicho cuidado.

Por otro lado, los cambios fisiológicos, emocionales y cognitivos en la tercera edad, como está ampliamente documentado, impactan en la autonomía del Adulto Mayor (AM) volviéndolo vulnerable y por consecuencia dependiente de una persona, principalmente de alguno de sus hijos o de un familiar.

De acuerdo a lo anterior, es importante plantear intervenciones enfocadas en que el adulto mayor se mantenga funcional el mayor tiempo posible para que este goce de una vejez saludable4. Aunque cada adulto mayor es diferente y tiene necesidades únicas, se ha estudiado que la actividad física, una alimentación balanceada y una red de apoyo adecuada, genera un impacto positivo en su salud.

Al respecto, existe una gran variedad de ejercicios como el Tai Chi, clasificado como un terapia alternativa o complementaria originaria de china ${ }^{5}$ considerada la actividad más adecuada para este 
grupo de edad, pues sus beneficios a la salud han sido ampliamente estudiados y para su práctica solo se requiere interés por aprenderlo.

Así, el objetivo de la presente revisión bibliográfica fue analizar la evidencia científica del uso del Tai Chi para conservar la salud del Adulto Mayor.

\section{METODOLOGÍA}

La revisión de la literatura se elaboró a partir de la información disponible en las bases de datos consultadas: Medline por Pubmed, Dialnet, SciELO, LILACS, ScienceDirect y Google Académico. Los términos empleados para la búsqueda fueron: Tai Chi Chuan, taiji quan, tai chi AND heatlh, tai chi improvements, Tai Chi Chuan AND adultos mayores, Taiji, Tai Ji, además se combinaron con el operador booleano AND. Se incluyeron artículos publicados en el periodo de 2013 al 2019.

Criterios de elegibilidad de los artículos: investigaciones realizadas en personas mayores de 60 años sanos o enfermos, artículos de acceso libre y texto completo; se incluyeron revisiones sistemáticas, revisiones bibliográficas, artículos en español, inglés y portugués.

Criterios de exclusión: artículos con antigüedad mayor de siete años.

Durante la búsqueda de la información se encontraron 2026 artículos en las diferentes bases de datos, se descartaron 1926 pues no cumplieron con los criterios de elegibilidad; 100 artículos fueron seleccionados por su relevancia; 56 carecían de datos importantes. Al final, esta revisión trabajó con 29 artículos de los cuales 16 se localizaron en Medline-PubMed, dos en SciELO, tres en Dialnet, dos en LILACS, dos en ScienceDirect y cuatro en Google Académico.

\section{RESULTADOS}

Investigadores de diversas partes del mundo interesados en el Tai Chi han observado los beneficios que esta terapia aporta a la salud de las personas; además de que dicha actividad puede realizarse bajo dos enfoques según el malestar: síntomas físicos y síntomas psicológicos/cognitivos.

\section{Sintomas físicos}

La mayor parte de los estudios realizados donde se ha aplicado Tai Chi están enfocados a la prevención de caídas (equilibrio y marcha). En 2014 Li F ${ }^{6}$ aplicó Tai Chi para mejorar los límites de estabilidad, (LOS) por su siglas en inglés, que es la capacidad de mantener el centro de gravedad dentro del límite de la base de apoyo. Después de 48 semanas de práctica se mejoraron esos límites de estabilidad punto final: $8.30 \%, \mathrm{p}<0.001$; velocidad de movimiento: 0.86 grados/segundos, $\mathrm{p}<0.001$; control de dirección: 6.79 \% of 100, $\mathrm{p}<0.001$. Por otro lado, Ory MG et al. ${ }^{7}$ utilizando la prueba Time Up and Go (TUG) la cual mide el tiempo en segundos requeridos para que los participantes se levanten de un sillón estándar, caminen a (su) ritmo típico o normal para alinearse en el piso a tres metros de distancia, girar, regresar y volver a sentarse; encontraron que en promedio, cuando se aplica Tai Chi, los participantes que completaron la prueba TUG en < 12 segundos se clasificaron como de bajo riesgo y los que tomaron 12 o más segundos se clasificaron como de alto riesgo. Las puntuaciones de la prueba TUG disminuyeron ( $\mathrm{p}$ < o.001) para todos los participantes; sin embargo, la disminución fue más notable entre los participantes de alto riesgo (la media disminuyó de 18.5 a 15.7 segundos).

En el mismo sentido, Hwang HF et al. ${ }^{8}$ encontraron en su estudio que el grupo que practicó Tai Chi Chuan tuvo significativamente menos probabilidades de experimentar una caída durante una intervención de seis meses (Tasa de Índice de Ratio [TIR]= 0.30, intervalo de confianza [IC] de $95 \%$ = 
0.15 - 0.60) y durante todo el periodo de estudio de 18 meses (TIR $=0.32$, IC del $95 \%=0.14-0.71$ ). Sin embargo, en el estudio que realizaron Sun W et al. ${ }^{9}$, identificaron que al comparar el Tai Chi contra realizar ejercicio (que consistía en caminar rápido), ambos mejoraban el control postural sobre una pierna. Por su parte, Mortazavi H et al. ${ }^{10}$, emplearon el Tai Chi para reducir el riesgo de caídas y encontraron que hubo una diferencia significativa entre los grupos al final de la octava y décima semana de práctica $(p<0.001$ ), esto se traduce en que, con el paso del tiempo, el Tai Chi redujo el riesgo de caídas.

En el caso de Manor B et al."1, utilizaron el Tai Chi para medir los efectos sobre la función física y la caminata de doble tarea en adultos mayores de 70 años que habitan casas de apoyo. Para ello, utilizaron la batería de rendimiento físico corto (SPPB) la cual es una prueba que incluye medidas de equilibrio de pie, velocidad de caminata de cuatro metros y la capacidad de levantarse de una silla cinco veces. Los hallazgos fueron que después de 12 semanas, el grupo de Tai Chi mostró una mayor mejora $(p=0.005$ ) en las puntuaciones de SPPB (línea de base $=8.1 \pm 2.9$, seguimiento= $9.0 \pm 2.6$ ) en comparación con los controles (línea de base $=8.2 \pm 2.6$, seguimiento= $8.2 \pm 2.6$ ). El Tai Chi también aumentó la velocidad de caminata normal y de doble tarea $(\mathrm{p}<0.001)$.

Silva-Zemanate MA et al. ${ }^{12}$, estudiaron la relación del Tai Chi con la capacidad funcional en un grupo de mujeres adultas mayores. Encontraron que en la Escala de Tinetti, referente a la prueba de balance la cual evalúa de manera objetiva y estandarizada el balance dinámico como estático, obtenida antes del programa de ejercicios, 59 \% de la población presentó una calificación normal y, al finalizar el programa de ejercicios, se incrementó en un $27 \%$. Con ello se alcanzó 86 \% de la población con calificación normal, siendo esta diferencia estadísticamente significativa al aplicar la prueba de $\operatorname{McNemar}(\mathrm{p}=0.031$ ). Por su parte, en la prueba de Romberg modificado, la cual consiste en evaluar la capacidad de la persona para permanecer de pie asumiendo distintas bases de sustentación, se identificó que, antes de la intervención con Tai Chi, 68 \% fue capaz de completar la prueba, después de la intervención 95 \% lo lograron, con un incremento de la capacidad de $27 \%$. En la prueba McNemar se advirtió que esta diferencia fue estadísticamente significativa $(p=0.031)$.

En el estudio de Song QH et al..$^{13}$, se comparó el Tai Chi con baile y caminata al paso de 12 meses. El grupo de Tai Chi mejoró de manera significativa de un día a otro en comparación con el del grupo de baile y el grupo de caminata $(\mathrm{p}<0.050 \mathrm{p}<0.01)$.

También se identificaron estudios que abordaron temas como la función cardiaca; por ejemplo el de Li Y et al. ${ }^{14}$, donde se investigó la relación entre la práctica del Tai Chi y los niveles séricos miR-24 y miR-155, biomarcadores que están asociados a cardiopatías. La primera (miR-24), está asociada con la mortalidad por insuficiencia cardiaca y la segunda (miR-155), es un biomarcador de la cardiopatía coronaria. Así, encontraron que después del entrenamiento de tres y seis meses, el Tai Chi redujo tanto los niveles séricos de miR-24 ( $p$ > 0.05) como los niveles séricos de miR-155 ( $>$ > 0.05). Por su parte, Ren X et al. ${ }^{15}$, estudiaron los efectos de la práctica del Tai Chi en pacientes con falla cardiaca, sus resultados fueron que la práctica habitual de esta terapia puede regular las cifras de la frecuencia del corazón; para dicho estudio emplearon un modelo de efectos aleatorios que calculó la Diferencia de Medias Ponderadas (DMP) como -2.52 lpm (IC de 95 \%: -3.49 a -1.55; p < 0.00), datos que resultaron estadísticamente significativos. La heterogeneidad fue $p=0.67$, también estudiaron la fracción de eyección del ventrículo izquierdo para lo cual utilizaron un modelo de efectos aleatorios que mostró la DMP de 9.94 \% (IC de 95 \%: 6.95 a 12.93; p < 0.00), lo cual tiene significancia estadística. Además, $\mathrm{p}<0.00$ para heterogeneidad (I2= $98 \%)$. 
Por otro lado, el dolor es un problema muy frecuente que deteriora la movilidad en las personas. Los estudios más realizados en este tema abordan la osteoartritis, el dolor lumbar, así como el dolor en diferentes sitios del cuerpo ${ }^{16,17}$. En el estudio de Hall A et al.$^{18}$, utilizaron la terapia de Tai Chi para aliviar alguna clase de dolor crónico. Se desarrollaron estudios con osteoartritis (80 \%), dolor de espalda (13 \%) y dolor de cabeza (7\%); el uso del enfoque GRADE (Grading of Recommendations, Assessment, Development and Evaluation), evidencia de calidad moderada de que el Tai Chi resultó más efectivo en comparación con ningún tratamiento o la atención habitual a corto plazo para el dolor (DME = -0.66 [IC de $95 \%=-0.85$ a -0.48 ]) y discapacidad (DME = -0.66 [IC de $95 \%$ = -0.85 a -0.46 ]).

Lee $\mathrm{YM}^{19}$ investigó los efectos de la práctica del Tai Chi sobre la presión arterial e identificó que la presión sistólica (PAS) mostró una diferencia significativa entre los grupos ( $\mathrm{p}=0.001$ ), la presión arterial diastólica (PAD) reportó una diferencia significativa entre los grupos ( $\mathrm{p}=0.001$ ). Un trabajo similar fue realizado por Teodoro $\mathrm{R}$ et al. ${ }^{20}$, en él estudiaron el efecto que tenía el Tai Chi sobre la presión arterial en un grupo de adultos mayores hipertensos y observaron que después de finalizar la sesión de Tai Chi, hubo una reducción significativa de la presión arterial media (PAM) desde el minuto 10, y una reducción significativa de la presión arterial sistólica (PAS) y diastólica (PAD) desde el minuto 20 hasta el minuto 60 después de la sesión ( $p<0.001$ ).

Incluso se encontró una investigación en el que se comparaba el Tai Chi contra la electro estimulación funcional. Hao Y et al. ${ }^{21}$, realizaron este estudio buscando evaluar el efecto del Tai Chi y la estimulación de los músculos de las extremidades inferiores en adultos mayores con insuficiencia cardiaca; para llevar a cabo su objetivo conformaron cuatro grupos de estudio: el primero recibió estimulación eléctrica funcional en los músculos de los miembros inferiores, el segundo practicó ejercicios de Tai Chi, el tercero correspondió al grupo control (no recibieron ningún tratamiento) y en el último grupo se aplicaron ambas terapias (electro estimulación y Tai Chi). Los investigadores observaron que, en comparación con el grupo de control, solo el grupo con electro estimulación funcional había aumentado la puntuación del Kansas City Cardiomyopathy Questionnaire (KCCQ), cuestionario que se utiliza para evaluar la calidad de vida física y social de los pacientes con alguna cardiopatía, entre más alto sea el puntaje mejor será el bienestar de la persona ( $p<0.0001, q$ = 9.06); en el grupo Tai Chi se observó una disminución de la frecuencia cardíaca en comparación al grupo control ( $p<0.0001, q$ = 5.72); se identificó una disminución en el consumo máximo de oxígeno durante una actividad física en los grupos de Tai Chi ( $p<0.0001, q=9.15)$ y en el grupo que recibieron ambas terapias ( $p<0.0001, q=10.69$ ), en los grupos control y electro estimulación no manifestaron cambios en esta variable.

En el estudio de Hägglund L et al. ${ }^{22}$ aplicaron la terapia del Tai Chi para evaluar el grado de fatiga en pacientes con insuficiencia cardiaca crónica y observaron que después de 16 semanas, el grupo de Tai Chi tendió a optar por una carga de entrenamiento más reducida y el grupo de control manifestó mayor fatiga mental. Los participantes en el grupo de entrenamiento calificaron un aumento de la fatiga general durante el seguimiento en comparación con la línea de base.

\section{Sintomas psicológicos y cognitivos}

El adulto mayor no solo atraviesa por cambios físicos y fisiológicos, también presenta cambios psicológicos y cognitivos, pues, con la experiencia de vida, su mente evoluciona.

En esta área se ha estudiado la influencia de la terapia en el estado de ánimo, estrés, depresión, ansiedad y enfado-tensión, memoria y atención; tal es la investigación de Orozco G et al. ${ }^{23}$ quienes estudiaron a 32 mujeres adultas mayores distribuidas en dos grupos. Como resultado, obtuvieron en 
la prueba neuropsicológica COGNISTAT diferencias significativas $(\mathrm{p}<0.05)$ entre los grupos. Las medias del grupo de Tai Chi en las dimensiones atención, memoria y construcción fueron mayores que las del grupo de control. Con esto se demuestra que en los grupos con práctica de Tai Chi mejoraron de manera significativa los síntomas psicológicos.

En otro estudio, Silva-Zemanate MA et al. ${ }^{12}$ utilizaron la práctica del Tai Chi para evaluar su efecto sobre la capacidad funcional de un grupo de adultos mayores. Posterior a la práctica aplicaron la prueba $t$ de Student pareada; los componentes con diferencia estadísticamente significativa fueron: memoria $(\mathrm{p}=0.047)$, atención-cálculo $(\mathrm{p}=0.012)$ y lenguaje $(\mathrm{p}=0.002)$. En la prueba total se obtuvo una media inicial de 26.4 puntos y se alcanzó una media final de 29.1 puntos, siendo esta diferencia estadísticamente significativa $(p=0.001)$. Para evaluar signos y síntomas de depresión utilizaron la Escala de Yesavage, en la prueba de McNemar no se encontró una diferencia significativa.

En el caso de Pan Z et al. ${ }^{24}$, estos autores emplearon la terapia de Tai Chi para estudiar la plasticidad neuronal de los practicantes. Identificaron que después de seis semanas la terapia contribuyó a mejorar la conectividad funcional en correlación con la mejora del rendimiento cognitivo. Como resultado, los participantes mostraron un mejor desempeño en el cálculo matemático después de la práctica de Tai Chi; así, el estudio sugiere que se obtiene un impacto inmediato sobre la actividad cerebral. Los grupos estudiados tuvieron una amplitud $\mathrm{P}_{3}$ significativamente más alta que la indicada en el grupo de adultos mayores sedentarios. Patrones de $\mathrm{P}_{3}$ similares entre adultos jóvenes y mayores que participan en ejercicios a largo plazo proporcionaron evidencia respecto a los beneficios del entrenamiento de resistencia y el ejercicio de Tai Chi sobre la función cognitiva.

Por otro lado, Wu MT et al..$^{25}$, emplearon el Tai Chi para mejorar el rendimiento de cambio de tarea en un grupo de adultos mayores. En su estudio observaron efectos significativos de interacción de grupo por tiempo sobre las medidas de activación del comportamiento y del cerebro. Específicamente, el grupo de Tai Chi mostró una mejor función física, disminución de los errores en el desempeño de cambio de tareas y aumento de la activación frontal superior izquierda para el contraste Switch con Non-switch antes y después de la intervención, mismos que no se observaron en el grupo control.

En la investigación de Siu MY y Lee $\mathrm{D}^{26}$, examinaron los efectos del Tai Chi sobre las funciones cognitivas generales. En este estudio los participantes del grupo obtuvieron puntuaciones significativamente mejores en la prueba de la versión China del Mini-Mental (en inglés CMMSE) ( $p=0.001$ ). Sin embargo, esos cambios en las puntuaciones no excedieron los límites establecidos del cambio mínimo detectable en el estudio, y no se pudo excluir la posibilidad de variación de la medición debido a errores.

Otro estudio realizado por Lam LCW et al..$^{27}$ compararon durante un año la efectividad del Tai Chi con el ejercicio de estiramiento y tonificación en el manejo del deterioro cognitivo y funcional en las personas mayores chinas con riesgo de deterioro cognitivo. Al finalizar el estudio, el primer grupo diagnosticado con demencia aumentó de $4.3 \%$ a 16.6 \%; en el grupo de Tai Chi se observó una disminución del riesgo de desarrollar demencia al cabo de un año (razón de posibilidades $[\mathrm{OR}]=0.28$, intervalo de confianza [IC] de $95 \%=0.05-0.92, p=0.064$ ).

En el mismo sentido, Liu S et al. ${ }^{28}$ utilizaron la terapia del Tai Chi para retrasar la atrofia de la materia gris y los vínculos de esta práctica con la estabilidad emocional. Al finalizar el estudio encontraron que el volumen de materia gris (GMV) del tálamo y el hipocampo fue mayor en el grupo de Tai Chi en comparación con el grupo de control. En particular, el GMV del tálamo se correlacionó positivamente tanto con el nivel de meditación como con la estabilidad emocional. 
En su estudio, Miller S y Taylor-Piliae $\mathrm{RE}^{29}$, compararon los procesos cognitivos y la función física relacionados con la conducción segura entre los adultos mayores practicantes de Tai Chi. Observaron que los participantes obtuvieron mejores resultados en diversas medidas cognitivas, incluida la prueba de escenas de conducción $(p<0.001, d=1.63$ ), la navegación por el laberinto ( $p=0.017, d=$ 0.27), la prueba de campo visual útil ( $p<0.001, r=0.15$ ), y en medidas físicas que incluyen la prueba de marcha rápida ( $\mathrm{p}<0.001, \mathrm{r}=0.20$ ) y la prueba de golpeteo del pie derecho, $(\mathrm{p}<0.001, \mathrm{r}=0.35)$.

En otras investigaciones que se han realizado alrededor de esta terapia, no han demostrado efecto alguno en la diabetes o en la capacidad aeróbica. Tal como lo demuestra el estudio que realizaron Hempel S et al..$^{\circ}$, en el cual encontraron que la evidencia existente no sugiere que el Tai Chi sea una terapia eficaz para el cuidado de la diabetes tipo 2. En cuanto a la capacidad aeróbica, encontraron que la evidencia existente no sugiere que la práctica regular del Tai Chi sea una forma efectiva de aumentar dicha capacidad.

También se encontraron estudios que sólo abordan el tema del estrés y la práctica del Tai Chi, tal como lo refleja el estudio de Marcelo-Donet AM et al. ${ }^{31}$. En dicho estudio midieron los niveles de estrés de dos grupos, la totalidad de adultos mayores que practican Tai Chi presentaron un nivel de estrés leve. En el grupo control, 65.3 \% demostraron tener nivel de estrés moderado, seguido de $26.5 \%$ con nivel de estrés alto. Las personas pertenecientes al grupo Tai Chi presentaron 19 puntos menos (IC 95 \% 17.3 - 22.5) en la evaluación de nivel de estrés independientemente al sexo y la edad.

Además, se encontraron otras investigaciones que abordaban el tema de la mejora en la calidad de vida al utilizar Tai Chi. Un ejemplo es la revisión de Orozco G et al. ${ }^{22}$; quienes reportaron la existencia de varios estudios que hablan de los beneficios que tiene esta práctica.

\section{CONCLUSIONES}

El Tai Chi es una práctica complementaria al tratamiento médico del paciente, por lo que esta actividad debe ser realizada de forma continua sin suspenderla. Por los beneficios encontrados en la literatura, se sugiere promover su práctica regular como una opción viable para mantener la salud del adulto mayor porque, además de ayudar a conservarla, es una opción fácil de aprender y de bajo costo. El personal de enfermería, como profesional de la salud, podría impulsar el autocuidado del adulto mayor, fomentando los beneficios de tal terapia por ejemplo ayuda al fortalecimiento del sistema musculo esquelético, la movilidad sin mucho dolor, marcha, equilibrio, sensación de bienestar, mejora de la memoria, disminución de depresión, ansiedad y estrés, entre otros.

Se consultó a profesores de amplia experiencia quienes coincidieron en que no existe un estilo mejor que otro, ni una forma específica que mejore la salud, ni un tiempo determinado de práctica para notar los beneficios. Partiendo de ello se recomienda que esta actividad se practique regularmente en sitios que estén cerca del hogar de los adultos mayores. Por último, es importante resaltar que el estilo y la forma en que se haya elegido practicar esta terapia no es lo más relevante, en cambio, sí lo es la correcta práctica del Tai Chi que integra tres aspectos relevantes: una mente calmada, así como la conciencia e intención en los movimientos.

\section{RESPONSABILIDADES ÉTICAS}

Protección de personas y animales. Al ser un estudio de revisión no se realizaron experimentos ni intervenciones.

Confidencialidad. Al ser una revisión, no se tuvo contacto con participantes. 
Conflicto de intereses. Los autores declaran no tener conflicto de intereses.

Financiamiento. Ninguno.

\section{REFERENCIAS}

1. Organización de las Naciones Unidas. La situación demográfica en el mundo, 2014. Informe conciso. Nueva York: ONU; 2014.

2. Kánter-Coronel IR. El envejecimiento en México, una visión demográfica. Pluralidad y Consenso. 2017; 7(33): 1-22. https://bit.ly/3bPdtGp

3. Organización Mundial de la Salud. Envejecimiento. Ginebra: OMS; 2015. https://bit.ly/3BZdBom

4. Organización Mundial de la Salud. ¿Qué repercusiones tiene el envejecimiento mundial en la salud pública?. Ginebra: OMS; 2011. https://bit.ly/31ybu7g

5. Organización Mundial de la Salud. Estrategia de la OMS sobre medicina tradicional 2014-2O23. Ginebra: OMS; 2013. https://bit.ly/3onSws2

6. Li F. The effects of Tai Ji Quan training on limits of stability in older adults. Clin Interv Aging. 2014; 4(9): 1261-8. https://doi.org/10.2147/CIA.S65823

7. Ory MG, Lee-Smith M, Parker EM, Jiang L, Chen S, Wilson AD, et al. Fall prevention in community settings: Results from implementing Tai Chi: Moving for Better Balance in three states. Front Public Heal. 2015; 2(258):1-6. https://doi.org/10.3389/fpubh.2014.00258

8. Hwang HF, Chen SJ, Lee-Hsieh J, Chien DK, Chen CY, Lin MR. Effects of home-based Tai Chi and lower extremity training and self-practice on falls and functional outcomes in older fallers from the emergency department-A randomized controlled trial. J Am Geriatr Soc. 2016; 64(3): 518-25. https://doi.org/10.1111/jgs.13952

9. Sun W, Wang L, Zhang C, Song Q, Gu H, Mao D. Detraining effects of regular Tai Chi exercise on postural control ability in older women: A randomized controlled trial. J Exerc Sci Fit. 2018; 16(2): 55-61. https://doi.org/10.1016/j.jesf.2018.06.003

10. Mortazavi H, Tabatabaeichehr M, Golestani A, Armat MR, Yousefi MR. The effect of Tai Chi exercise on the risk and fear of falling in older adults: A randomized clinical trial. Mater Sociomed. 2018; 30(1): 38-42. https://doi.org/10.5455/msm.2018.30.38-42

11. Manor B, Lough M, Gagnon MM, Cupples A, Wayne PM, Lipsitz LA. Functional benefits of Tai Chi training in senior housing facilities. J Am Geriatr Soc. 2014; 62(8): 1484-9.

https://doi.org/10.1111/jgs.12946

12. Silva-Zemanate MA, Pérez-Solarte SL, Fernández-Cerón MI, Tovar-Ruíz LÁ. Efectos del Tai Chi en la capacidad funcional de un grupo de mujeres ancianas. Rev. Cienc. Salud. 2014; 12(3): 353-69.

https://doi.org/10.12804/revsalud12.03.2014.05

13. Song QH, Zhang QH, Xu RM, Ma M, Zhao XP, Shen GQ, et al. Effect of Tai Chi exercise on lower limb muscle strength, bone mineral density and balance function of elderly women. Int J Clin Exp Med. 2014; 7(6): 1569-76. https://bit.ly/3qk6ZYj

14. Li Y, Zhang H, Wang Y. Tai Chi ameliorates coronary heart disease by affecting serum levels of miR-24 and miR-155. Front Physiol. 2019; 10(587): 1-9. https://doi.org/10.3389/fphys.2019.00587

15. Ren X, Li Y, Yang X, Li J, Li H, Yuan Z, et al. The effects of Tai Chi training in patients with heart failure: A systematic review and meta-analysis. Front Physiol. 2017; 8(989): 1-13.

https://doi.org/10.3389/fphys.2017.00989 
16. Kong LJ, Lauche R, Klose P, Bu JH, Yang XC, Guo CQ, et al. Tai Chi for chronic pain conditions: A systematic review and meta-analysis of randomized controlled trials. Sci Rep. 2016; 6: 1-9.

https://doi.org/10.1038/srep25325

17. You T, Ogawa EF, Thapa S, Cai Y, Zhang H, Nagae S, et al. Tai Chi for older adults with chronic multisite pain: A randomized controlled pilot study. Aging Clin Exp Res. 2018; 30(11): 1335-43.

https://doi.org/10.1007/s40520-018-0922-0

18. Hall A, Copsey B, Richmond H, Thompson J, Ferreira M, Latimer J, et al. Effectiveness of Tai Chi for chronic musculoskeletal pain conditions: Updated systematic review and meta-analysis. Phys Ther. 2017; 97(2): 227-38. https://doi.org/10.2522/ptj.20160246

19. Lee YM. The effects of Tai Chi on waist circumference and blood pressure in the elderly. J Phys Ther Sci. 2017; 29(1): 172-5. https://doi.org/10.1589/jpts.29.172

20. Teodoro RST, Gregorio-Joaquim A, Da Silva-Xavier JJ, Marques-Miguel Suen V, Marchi-Alves Ancheschi LM. Sessão de Tai Chi Chuan promove hipotensão pós-exercício e redução da sobrecarga cardiovascular em idosos hipertensos. Medicina. 2017; 50(5): 297-306.

https://doi.org/10.11606/issn.2176-7262.v50i5p297-306

21. Hao Y, Zhang L, Zhang Z, Chen L, He N, Zhu S. Tai Chi exercise and functional electrical stimulation of lower limb muscles for rehabilitation in older adults with chronic systolic heart failure: A non-randomized clinical trial. Braz. J. Med. Biol. Res. 2019; 52(12): 1-7.

http://dx.doi.org/10.159o/1414-431X20198786

22. Hägglund L, Boman K, Brännström M. A mixed methods study of Tai Chi exercise for patients with chronic heart failure aged 70 years and older. Nurs Open. 2018; 5(2): 176-85.

https://doi.org/10.1002/nop2.127

23. Orozco-Calderón G, Anaya-Chávez M, Santiago-Vite J, García Viedma MR. Cognición, actividades de la vida diaria y variables psicológicas mujeres adultas mayores practicantes de Tai Chi Chuan (Yang). Retos. 2016; 30: 222-5. https://bit.ly/3oddLg3

24. Pan Z, Su X, Fang O, Hou L, Lee Y, Chen CC, et al. The effects of Tai Chi intervention on healthy elderly by means of neuroimaging and EEG: A systematic review. Front Aging Neurosci. 2018; 10(110). https://doi.org/10.3389/fnagi.2018.00110

25. Wu MT, Tang PF, Goh JOS, Chou TL, Chang YK, Hsu YC, et al. Task-switching performance improvements after Tai Chi Chuan training are associated with greater prefrontal activation in older adults. Front Aging Neurosci. 2018; 10(280): 1-17. https://doi.org/10.3389/fnagi.2018.00280

26. Siu MY, Lee DTF. Effects of Tai Chi on cognition and instrumental activities of daily living in community dwelling older people with mild cognitive impairment. BMC Geriatr. 2018; 18(1): 1-10. https://doi.org/10.1186/s12877-018-0720-8

27. Lam LCW, Chan WM, Kwok TCY, Chiu HFK. Effectiveness of Tai Chi in maintenance of cognitive and functional abilities in mild cognitive impairment: A randomised controlled trial. Hong Kong Med J. 2014; 2O(Suppl 3): S2O-3. https://bit.ly/3koSNK6

28. Liu S, Li L, Liu Z, Guo X. Long-term Tai Chi experience promotes emotional stability and slows gray matter atrophy for elders. Front Psychol. 2019; 10(91): 1-11. https://doi.org/10.3389/fpsyg.2019.00091

29. Miller S, Taylor-Piliae RE. The association between Tai Chi exercise and safe driving performance among older adults: An observational study. J Sport Health Sci. 2018; 7(1): 83-94.

https://doi.org/10.1016/j.jshs.2017.01.013 
30. Hempel S, Taylor SL, Solloway MR, Miake-Lye IM, Beroes JM, Shanman R, et al. Evidence map of Tai Chi. Washington D.C.: Department of Veterans Affairs (US); 2014. https://bit.ly/30g61cX

31. Marcelo-Donet AM, Valderrama-Rojas GM, León-Castillo MR. Práctica de Taichí y nivel de estrés en adultos mayores en un centro de atención de medicina complementaria. Rev. peru. med. integr. 2016; 1(4): 25-30. https://doi.org/10.26722/rpmi.2016.14.32

32. Orozco-Calderón G, Santiago-Vite J, Anaya-Chávez M, Guerrero S. Efectos psicológicos y cognitivos de la práctica de Tai Chi en adultos mayores. RU-Iztacala. 2016; 19(3): 858-76.

https://bit.ly/3ogfigW

33. Carrillo J, Gómez-López M, Vicente G. El Taiji Quan y Qigong en la promoción de la salud y en el afrontamiento del estrés. Sport TK. 2017; 6(2): 59-74. https://doi.org/10.6018/300401

34. Moura-Pereira M, Carolino-Souza V, Paula AP, Franco-Moraes C, Toledo-Nóbrega O, Gomes L. Tai Chi Chuan improves functionality and quality of life in elderly men with low bone mineral density. Geriatr Gerontol Aging. 2017; 11(4): 174-81. https://doi.org/10.5327/Z2447-211520171700036 